\title{
Noncommutative Gravity
}

\author{
E. Harikumar and Victor O. Rivelles \\ Instituto de Física, \\ Universidade de São Paulo \\ Caixa Postal 66318, 05315-970, \\ São Paulo, SP, Brazil \\ E-mail: hari@fma.if.usp.br and rivelles@fma.if.usp.br
}

\begin{abstract}
We consider simple extensions of noncommutativity from flat to curved spacetime. One possibility is to have a generalization of the Moyal product with a covariantly constant noncommutative tensor $\theta^{\mu \nu}$. In this case the spacetime symmetry is restricted to volume preserving diffeomorphisms which also preserve $\theta^{\mu \nu}$. Another possibility is an extension of the Kontsevich product to curved spacetime. In both cases the noncommutative product is nonassociative. We find the order $\theta^{2}$ noncommutative correction to the Newtonian potential in the case of a covariantly constant $\theta^{\mu \nu}$. It is still of the form $1 / r$ plus an angle dependent piece. The coupling to matter gives rise to a propagator which is $\theta$ dependent.
\end{abstract}




\section{INTRODUCTION}

Noncommuting coordinates were proposed a long time ago as a generalization of the phase space of quantum mechanics [1]. The idea was resurrected in the string theory context when a decoupling limit was found that yields a noncommutative gauge theory induced by the Moyal product 2]. From then on its properties were studied in detail and a large amount of information on noncommutative field theories were gained [3].

The main characteristic of noncommutative field theories is that it incorporates non-local effects in a controllable way. This is reminiscent of its stringy origin where the gravitational sector was decoupled but still left some traces through the noncommutativity. In the quantum theory particles present a kind of dipole structure [4] while in quantum field theory there appears a mixing of ultraviolet and infrared divergences [5]. Another interesting effect is that matter fields can feel noncommutativity as a background gravitational field [6].

So there is a good understanding of noncommutative effects in matter and gauge fields in flat spacetime. The next step is to incorporate gravity and thus consider curved spacetimes. The main trouble we have to face now is how to deal with the noncommutative parameter which is usually taken to be constant in flat spacetime. This gave rise to a large amount of work to study deformations of general relativity [7, 8, 9, 10]. Usually noncommutative deformations of gravity lead to a complex metric and tangent space groups larger than the Lorentz group 9]. Another approach is to replace the diffeomorphism invariance of general relativity by a twisted version of it [10]. Both of these approaches lead to gravitational theories which are not simple extensions of general relativity. An attempt in this direction considers that the enveloping algebra of the Lorentz group is gauged and volume preserving diffeomorphisms are used instead of general coordinate transformations. Gravitational theories based on volume preserving diffeomorphisms are usually known as unimodular gravity theories [11] and a noncommutative deformation of it is proposed in [12].

More recently an attempt to derive noncommutative gravity from string theory was presented [13]. The dynamics of closed strings in the presence of a constant B-field induces a gravitational action in the next-to-leading order in the Seiberg-Witten limit 2] and some of the three gravitons interaction vertices are derived. It happens that these vertices can not be obtained from an action written only with Moyal products and the authors claim that this is due to the fact that the Moyal product changes under a space-time diffeomorphism.

In this paper we present an alternative approach. Instead of implementing diffeomorphisms in the noncommutative setting we extend the properties of noncommutativity in flat spacetime to curved spacetime in a way that is as simple as possible. In flat spacetime we usually assume that the noncommutative parameter $\theta^{\mu \nu}$ is constant and its infinitesimal functional variation vanishes. As we shall see, we need only to assume that the functional variation of $\theta^{\mu \nu}$ is zero since its constancy will be a consequence of preserving translation invariance. We then assume the same condition in curved spacetime. Now we obtain that $\theta^{\mu \nu}$ is a covariantly constant tensor [14] and that the symmetries of spacetime reduce to volume preserving diffeomorphisms which also preserve $\theta^{\mu \nu}$. An alternative extension to curved spacetime is to consider the flat spacetime Kontsevich product where $\theta^{\mu \nu}$ is no longer constant but satisfies the Jacobi identities. This condition can easily be extended to curved spacetime.

In our setting noncommutativity can couple to the spacetime geometric tensors. It also manifests itself through the coupling to matter by extensions of the Moyal and Kontsevich products to curved spacetime. While in flat spacetime both products are associative, no 
simple extension of them to curved spacetime is found which also preserves associativity.

We study the consequences of such a proposal finding noncommutative corrections to the Newtonian potential in the case where $\theta^{\mu \nu}$ is covariantly constant. The correction still has the Newtonian form $1 / r$ but with an effective Newton constant. In addition, there is a piece which has an angular dependence.

Section II discusses how we can extend the properties of the noncommutativity parameter $\theta^{\mu \nu}$ from flat spacetime to curved spacetime. We consider the case of constant $\theta^{\mu \nu}$ used in the Moyal product and the case of a non constant $\theta^{\mu \nu}$ used in the Kontsevich product. In Section III we discuss the coupling to matter and how the nonassociativity emerges. Section IV is devoted to the study of the coupling of noncommutativity to gravity in the case where $\theta^{\mu \nu}$ is covariantly constant. In the next Section we discuss the linearized limit to find the noncommutative correction to the Newtonian potential. Finally, the last Section is devoted to some comments and conclusions.

\section{NONCOMMUTATIVITY IN FLAT AND CURVED SPACETIME}

In flat spacetime noncommutativity is described by a constant antisymmetric matrix $\theta^{\mu \nu}$ which has the same value in all inertial frames and leads to a breakdown of Lorentz invariance. Let us consider this in more detail in order to be able to find which properties from flat spacetime can be generalized to a curved one. Let us promote $\theta^{\mu \nu}$ to a tensor under Lorentz transformations and perform a general coordinate transformation with infinitesimal parameter $\xi^{\mu}$

$$
\delta \theta^{\mu \nu}=\xi^{\lambda} \partial_{\lambda} \theta^{\mu \nu}-\partial_{\lambda} \xi^{\mu} \theta^{\lambda \nu}+\partial_{\lambda} \xi^{\nu} \theta^{\lambda \mu} .
$$

Recall that $\delta \theta^{\mu \nu}$ stands for a functional variation of $\theta^{\mu \nu}$ at the same point and we are going to require its vanishing. For a rigid translation $\xi^{\mu}$ is constant and we get $\partial_{\lambda} \theta^{\mu \nu}=0$ so that all components of $\theta^{\mu \nu}$ are constant. For a Lorentz transformation $\xi^{\mu}=\Lambda^{\mu}{ }_{\nu} x^{\nu}$ we get

$$
\Lambda_{\lambda}^{\mu} \theta^{\lambda \nu}-\Lambda_{\lambda}^{\nu} \theta^{\lambda \mu}=0 .
$$

For instance, if the only nonvanishing component of $\theta^{\mu \nu}$ is $\theta^{12}$ then Lorentz boosts in 3direction and rotations in the $1-2$ plane are still preserved as well as translations in any direction.

There is one more transformation which solves $\delta \theta^{\mu \nu}=0$ and that is

$$
\xi^{\mu}=\theta^{\mu \nu} \partial_{\nu} \xi
$$

where $\xi$ is a scalar function. Notice that $\partial_{\mu} \xi^{\mu}=0$. If we work with curvilinear coordinates the volume element $d^{4} x$ is a scalar density of weight 1 so that it is invariant under an infinitesimal transformation satisfying $\partial_{\mu} \xi^{\mu}=0$. Then this is a volume preserving transformation. However, transformation (2.3) is a specific volume preserving transformation. Since the commutator of two infinitesimal transformations with parameters $\xi_{1}^{\mu}$ and $\xi_{2}^{\mu}$ gives a transformation with parameter $\xi_{3}^{\mu}=\xi_{1}^{\lambda} \partial_{\lambda} \xi_{2}^{\mu}-\xi_{2}^{\lambda} \partial_{\lambda} \xi_{1}^{\mu}$, for the particular case (2.3) we get

$$
\xi_{3}^{\mu}=\theta^{\mu \nu} \partial_{\nu} \xi_{3}, \quad \xi_{3}=-\theta^{\mu \nu} \partial_{\mu} \xi_{1} \partial_{\nu} \xi_{2},
$$

so that they form a subgroup of the volume preserving transformations. Since $\theta^{\mu \nu}$ is preserved by these transformations we get a sympletic subgroup of the volume preserving diffeomorphisms [15]. These residual symmetries are not useful in flat spacetime, even though 
there are some attempts to use them [16]. However, they will be useful in curved spacetime as it will be shown. Hence, the requirement that $\theta^{\mu \nu}$ be a tensor whose functional variation under Poincaré transformations vanishes, seems to be a good starting point for generalization. Notice that $\delta \theta^{\mu \nu}=0$ leads not only to a condition on $\theta^{\mu \nu}$ but it also implies that Lorentz transformations in some directions are broken.

Consider now a spacetime whose Riemannian geometry is associated to a metric tensor $g_{\mu \nu}$. Under an infinitesimal diffeomorphism an antisymmetric tensor $\theta^{\mu \nu}$ transforms as

$$
\delta \theta^{\mu \nu}=\xi^{\lambda} D_{\lambda} \theta^{\mu \nu}-D_{\lambda} \xi^{\mu} \theta^{\lambda \nu}+D_{\lambda} \xi^{\nu} \theta^{\lambda \mu}
$$

We now impose that $\delta \theta^{\mu \nu}=0$, as in flat spacetime, and we will solve this equation as constraints on $\theta^{\mu \nu}$ and on the diffeomorphisms. A solution which generalizes the constancy of $\theta^{\mu \nu}$ in flat spacetime is that it is covariantly constant

$$
D_{\lambda} \theta^{\mu \nu}=0
$$

which implies that

$$
D_{\lambda} \xi^{\mu} \theta^{\lambda \nu}-D_{\lambda} \xi^{\nu} \theta^{\lambda \mu}=0 .
$$

The solution to this last equation is simply (2.3). Notice that $D_{\mu} \xi^{\mu}=0$ so that there is a residual symmetry similar to what happens in flat spacetime. We are left with the sympletic subgroup of the volume preserving diffeomorphisms which also preserves a covariantly constant $\theta^{\mu \nu}$. We would like to point out that this symmetry also appears in another formulation of noncommutative gravity which makes use of noncommuting coordinates 12].

Another starting point would be the use of the Kontsevich product 17] instead of the Moyal product. In this case $\theta^{\mu \nu}$ is a tensor even in flat spacetime and satisfies the Jacobi identities

$$
\theta^{\lambda \mu} \partial_{\lambda} \theta^{\nu \rho}+\theta^{\lambda \nu} \partial_{\lambda} \theta^{\rho \mu}+\theta^{\lambda \rho} \partial_{\lambda} \theta^{\mu \nu}=0,
$$

which turns the Kontsevich product into an associative product. What is remarkable is that besides respecting Poincaré symmetry $\theta^{\mu \nu}$ is left invariant by the sympletic subgroup of volume preserving diffeomorphisms for $\theta^{\mu \nu}$ satisfying (2.8). So the Kontsevich product also allows a volume preserving diffeomorphism if $\theta^{\mu \nu}$ is form invariant. Even though providing an alternative way to implement noncommutativity in flat spacetime its consequences in quantum field theories are far less understood than in the Moyal case [18].

Using the Kontsevich product we no longer have the condition $\delta \theta^{\mu \nu}=0$ as in flat spacetime. The generalization to curved spacetime is straightforward since (2.8) is covariantized as

$$
\theta^{\lambda \mu} D_{\lambda} \theta^{\nu \rho}+\theta^{\lambda \nu} D_{\lambda} \theta^{\rho \mu}+\theta^{\lambda \rho} D_{\lambda} \theta^{\mu \nu}=0 .
$$

This condition now replaces (2.6) and we find no further condition on general diffeomorphisms. As in the flat spacetime case we can require that $\theta^{\mu \nu}$ is form invariant and we find that volume preserving diffeomorphisms are allowed if use is made of (2.9).

\section{COUPLING TO MATTER}

We assume that the geometric quantities are unaffected by noncommutativity so that we can define the Christoffel symbol, covariant derivatives, the Riemann tensor, and so on in the usual way. Noncommutativity can appear as in flat spacetime, by replacing the ordinary 
product by a Moyal product where the ordinary derivatives have been replaced by covariant ones. For two tensors $T_{\mu_{1} \ldots}$ and $S_{\nu_{1} \ldots}$ we write formally

$$
T_{\mu_{1} \ldots}(x) \star S_{\nu_{1} \ldots}(x)=\left.e^{\frac{i}{2} \theta^{\alpha \beta} D_{\alpha}(x) D_{\beta}(y)} T_{\mu_{1} \ldots}(x) S_{\nu_{1} \ldots}(y)\right|_{y=x} .
$$

This is a formal definition because the covariant derivatives do not commute and we must specify a precise ordering for second and higher order terms in an expansion in $\theta$.

One of the main properties of the Moyal product in flat spacetime is associativity so we would like to retain it in curved spacetime. Unfortunately we could not find any ordering which makes the Moyal product in (3.1) associative. For instance, defining (3.1) up to second order in $\theta$ as

$$
T \star S=T S+\frac{i}{2} \theta^{\alpha \beta} D_{\alpha} T D_{\beta} S-\frac{1}{8} \theta^{\alpha_{1} \beta_{1}} \theta^{\alpha_{2} \beta_{2}} D_{\alpha_{1}} D_{\alpha_{2}} T D_{\beta_{1}} D_{\beta_{2}} S
$$

(tensorial indices were omitted) we find that

$$
\begin{aligned}
(T \star S) \star U & -T \star(S \star U)=-\frac{1}{4} \theta^{\alpha_{1} \beta_{1}} \theta^{\alpha_{2} \beta_{2}}\left(-\frac{1}{2} D_{\alpha_{1}} T D_{\alpha_{2}} S\left[D_{\beta_{1}}, D_{\beta_{2}}\right] U\right. \\
& \left.+\frac{1}{2}\left[D_{\beta_{1}}, D_{\beta_{2}}\right] T D_{\alpha_{2}} S D_{\alpha_{1}} U+D_{\alpha_{1}} T\left[D_{\beta_{1}}, D_{\beta_{2}}\right] S D_{\alpha_{2}} U\right) .
\end{aligned}
$$

Even if we change the ordering in the quadratic term in $\theta$ in (3.2) we were not able to recover associativity. For instance, if we take the symmetric part in $\alpha_{1} \alpha_{2}$ and also in $\beta_{1} \beta_{2}$ we still get the same result (3.3). Other modifications of (3.2), like having terms with one derivative in $\mathrm{S}$ and three derivatives in $\mathrm{T}$, were also tried without success.

Another possibility would be to consider the Kontsevich product which in flat spacetime is associative if (2.8) holds. In curved spacetime we generalize the Kontsevich product by replacing ordinary derivatives by covariant ones and up to second order in $\theta$ we obtain

$$
\begin{aligned}
T \star S & =T S+\frac{i}{2} \theta^{\alpha \beta} D_{\alpha} T D_{\beta} S-\frac{1}{8} \theta^{\alpha_{1} \beta_{1}} \theta^{\alpha_{2} \beta_{2}} D_{\alpha_{1}} D_{\alpha_{2}} T D_{\beta_{1}} D_{\beta_{2}} S- \\
& -\frac{1}{12} \theta^{\alpha_{1} \beta_{1}} D_{\beta_{1}} \theta^{\alpha_{2} \beta_{2}}\left(D_{\alpha_{1}} D_{\alpha_{2}} T D_{\beta_{2}} S+D_{\beta_{2}} T D_{\alpha_{1}} D_{\alpha_{2}} S\right) .
\end{aligned}
$$

An explicit calculation shows that if we use (2.9) one still gets (3.3) so the proposed extension of the Kontsevich product to curved spacetime is no longer associative.

The origin of nonassociativity can be traced to string theory. When we consider D-branes in curved backgrounds it is known that a nonassociative star product is needed [19]. There is nothing wrong with nonassociative products. It just turns life more difficult. We have to be extremely careful when writing products since the order now must be clearly specified.

In flat spacetime the Moyal product of two fields gives the ordinary product of them plus total derivative terms. When writing an action, terms in $\theta$ can then be disregarded. In curved spacetime this property no longer holds for the Moyal product (3.2). It gives a total (covariant) derivative term plus $\theta$ contributions involving curvature terms. So when writing an action which is quadratic in the fields the Moyal product gives non trivial contributions involving $\theta$. For instance, we can write the action for a scalar field as

$$
S=\frac{1}{2} \int \sqrt{-g} d^{4} x\left[g^{\mu \nu} \partial_{\mu} \Phi \star \partial_{\nu} \Phi-m^{2} \Phi \star \Phi\right],
$$


and expanding as in (3.2) to second order in $\theta$ we find

$$
\begin{array}{r}
S=\frac{1}{2} \int \sqrt{-g} d^{4} x\left[g^{\mu \nu} \partial_{\mu} \Phi \partial_{\nu} \Phi-\frac{1}{8} g^{\mu \nu} \theta^{\alpha_{1} \beta_{1}} \theta^{\alpha_{2} \beta_{2}} D_{\alpha_{1}} D_{\alpha_{2}} \partial_{\mu} \Phi D_{\beta_{1}} D_{\beta_{2}} \partial_{\nu} \Phi\right. \\
\left.-m^{2} \Phi \Phi+\frac{m^{2}}{8} \theta^{\alpha_{1} \beta_{1}} \theta^{\alpha_{2} \beta_{2}} D_{\alpha_{1}} \partial_{\alpha_{2}} \Phi D_{\beta_{1}} \partial_{\beta_{2}} \Phi\right] .
\end{array}
$$

Notice that in flat spacetime the propagators receive no $\theta$ correction because the quadratic terms in the action have no $\theta$ dependence. The noncommutative contribution appears exclusively at the interaction vertices. In curved spacetime we find that the propagators are now $\theta$ dependent and are modified by noncommutativity. This is in striking difference with flat spacetime.

\section{NONCOMMUTATIVE GRAVITY}

Since we are leaving the geometry untouched by noncommutativity the only way to study its effect in a purely gravitational context is through the coupling to the geometric tensors. Recall that $\theta^{\mu \nu}$ is a tensor but has no dynamics. It is constrained either to be covariantly constant (2.6) or by (2.9). For simplicity we will consider the case where $\theta^{\mu \nu}$ is covariantly constant and consider only the lowest order couplings in $\theta^{\mu \nu}$. Then it couples to the scalar curvature as $\theta^{\mu \nu} \theta_{\mu \nu} R$, to the Ricci tensor as $\theta^{\mu \nu} \theta_{\nu}{ }^{\lambda} R_{\mu \lambda}$, and to the Riemann tensor as $\theta^{\mu \nu} \theta^{\alpha}{ }_{\beta} R_{\mu \nu \alpha}{ }^{\beta}$ (the coupling $\theta^{\mu \nu} \theta^{\alpha}{ }_{\beta} R_{\mu \alpha \nu}{ }^{\beta}$ reduces to the former one). Since noncommutativity is very small we can assume a perturbative scheme for $\theta^{\mu \nu}$ and look for corrections to general relativity. If we consider only vacuum solutions of Einstein equations then the first two couplings are not relevant and only the third one should be regarded.

Then we start with the Einstein-Hilbert action plus the noncommutative contribution

$$
S_{N C}=\frac{1}{16 \pi} \int d^{4} x \sqrt{-g} \theta^{\mu \nu} \theta^{\alpha}{ }_{\beta} R_{\mu \nu \alpha}{ }^{\beta} .
$$

Notice that we need a dimensionfull coupling constant with dimension $L^{-6}$ which can be absorbed in $\theta$, so that it has dimension $L^{-1}$ instead of the usual dimension $L^{2}$. There is also a global sign which can not be absorbed in $\theta$ but which can be changed by $\theta^{2} \rightarrow-\theta^{2}$.

As discussed in section II, the introduction of a covariantly constant $\theta^{\mu \nu}$ reduces the diffeomorphism transformations to a subgroup of volume preserving transformations. As remarked in the introduction, gravity theories based on volume preserving transformations are known as unimodular gravity theories [11]. Since $D_{\mu} \xi^{\mu}=0$ for a volume preserving transformation then the determinant of the metric, which transforms as a scalar density of weight -2 , is invariant so that $\operatorname{det} g$ is constant and usually taken to be minus one. Then we have a theory with an absolute spacetime volume element, the modulus. Unimodular gravity has many interesting properties. The cosmological constant appears as an integration constant and the action has a finite polynomial form in the metric [20]. Since we can take any solution of general relativity and go to a frame where $\operatorname{det} g=-1$ there is no physical difference between both gravity theories [11, 22]. In our case, the Einstein-Hilbert action is invariant under general diffeomorphisms but $S_{N C}$ (4.1) is invariant under the restricted class of volume preserving diffeomorphisms.

The equations of motion we obtain are then

$$
\frac{1}{G}\left(R_{\mu \nu}-\frac{1}{2} g_{\mu \nu} R\right)-\frac{1}{2} g_{\mu \nu} \theta^{\rho \lambda} \theta^{\alpha}{ }_{\beta} R_{\rho \lambda \alpha}{ }^{\beta}-\frac{1}{2} \theta^{\alpha \beta} \theta_{\mu \gamma} R_{\alpha \beta \nu}{ }^{\gamma}-\frac{1}{2} \theta^{\alpha \beta} \theta_{\nu \gamma} R_{\alpha \beta \mu}{ }^{\gamma}=0,
$$


and since the Einstein tensor is covariantly conserved this implies that

$$
3 \theta^{\alpha \beta} \theta^{\rho \sigma} D_{\alpha} R_{\mu \beta \rho \sigma}-2 \theta^{\alpha \beta} \theta^{\rho}{ }_{\mu} D_{\alpha} R_{\beta \rho}=0 .
$$

As we will see, this last equation, together with (2.6), puts stringent constraints on the components of $\theta^{\mu \nu}$.

\section{LINEARIZED NONCOMMUTATIVE GRAVITY}

To study the effects of noncommutativity on the gravitational field we will consider the linearized approximation and obtain corrections to the Newtonian potential. As usual we expand the metric around flat spacetime, $g_{\mu \nu}=\eta_{\mu \nu}+h_{\mu \nu}$. The linearization of (4.2) gives

$$
\begin{aligned}
& \frac{1}{G}\left[\square h_{\mu \nu}+\partial_{\mu} \partial_{\nu} h_{\rho}{ }^{\rho}-\partial^{\rho} \partial_{\mu} h_{\nu \rho}-\partial^{\rho} \partial_{\nu} h_{\mu \rho}-\eta_{\mu \nu}\left(\square h_{\rho}{ }^{\rho}-\partial^{\rho} \partial^{\sigma} h_{\rho \sigma}\right)\right] \\
+ & \theta^{\alpha \beta} \theta_{\mu \gamma}\left(\partial_{\nu} \partial_{\alpha} h_{\beta}^{\gamma}-\partial^{\gamma} \partial_{\alpha} h_{\beta \nu}\right)+\theta^{\alpha \beta} \theta_{\nu \gamma}\left(\partial_{\mu} \partial_{\alpha} h_{\beta}^{\gamma}-\partial^{\gamma} \partial_{\alpha} h_{\beta \mu}\right)+2 \eta_{\mu \nu} \theta^{\alpha \beta} \theta_{\sigma}{ }_{\sigma} \partial_{\rho} \partial_{\alpha} h_{\beta}{ }^{\sigma}=0,
\end{aligned}
$$

while (2.6) yields

$$
\partial_{\mu} \theta^{\alpha \beta}+\frac{1}{2}\left(\partial_{\mu} h_{\lambda}{ }^{[\alpha}+\partial_{\lambda} h_{\mu}^{[\alpha}-\partial^{[\alpha} h_{\mu \lambda}\right) \theta^{\lambda \beta]}=0 .
$$

In unimodular gravity we have $\operatorname{det} g_{\mu \nu}=-1$ which, at the linearized level, implies that $h_{\mu \nu}$ is traceless. Then (5.1) gives

$$
\square h_{\mu \nu}-\partial^{\rho} \partial_{\mu} h_{\nu \rho}-\partial^{\rho} \partial_{\nu} h_{\mu \rho}+\eta_{\mu \nu} \partial^{\rho} \partial^{\sigma} h_{\rho \sigma}=\mathcal{O}\left(\theta^{2}\right),
$$

and contracting this equation with $\eta^{\mu \nu}$ we get

$$
\partial^{\mu} \partial^{\nu} h_{\mu \nu}=\mathcal{O}\left(\theta^{2}\right)
$$

so that (15.3) reduces to

$$
\square h_{\mu \nu}-\partial^{\rho} \partial_{\mu} h_{\nu \rho}-\partial^{\rho} \partial_{\nu} h_{\mu \rho}=\mathcal{O}\left(\theta^{2}\right) .
$$

The first step is to find the solution for the Newtonian potential in unimodular gravity. We can either take the solution in Cartesian coordinates

$$
g_{\mu \nu}=\eta_{\mu \nu}+\delta_{\mu \nu} h, \quad h=\frac{-2 G M}{r},
$$

where $r=\sqrt{x^{2}+y^{2}+z^{2}}$, and perform a coordinate transformation so that det $g_{\mu \nu}=-1$, or solve (5.4) and (5.5) directly with a vanishing rhs. The coordinate transformation is given by

$$
x^{\prime 0}=x^{0}, \quad x^{i}=\left(1-\frac{h}{2}\right) x^{i} .
$$

Anyway, we find that the Newtonian potential in Cartesian coordinates is given by

$$
h_{00}=h, \quad h_{0 i}=0, \quad h_{i j}=n^{i} n^{j} h,
$$

where $n^{i}=x^{i} / r$ is the radial unit vector. As required, $h_{\mu \nu}$ is traceless and satisfies $\partial^{\mu} \partial^{\nu} h_{\mu \nu}=$ 0 . 
Having (5.8) as a solution of (5.1) to order zero in $\theta$ and first order in $G M$ we can now solve (5.2) perturbatively in $\theta$ and $G M$. Then $\theta^{\mu \nu}$ must satisfy $\partial_{\mu} \theta^{\alpha \beta}=0$, that is, $\theta^{\mu \nu}$ is constant.

We can now find the $\theta$ dependent terms which contribute to $g_{\mu \nu}$. We write $g_{\mu \nu}=\eta_{\mu \nu}+$ $h_{\mu \nu}+\bar{h}_{\mu \nu}$ where $h_{\mu \nu}$ is given by (5.8) and $\bar{h}_{\mu \nu}$ is of order $\theta^{2}$ and traceless. We then find that (5.3) reduces to

$$
\begin{aligned}
& \frac{1}{G}\left(\square \bar{h}_{\mu \nu}-\partial^{\rho} \partial_{\mu} \bar{h}_{\nu \rho}-\partial^{\rho} \partial_{\nu} \bar{h}_{\mu \rho}+\eta_{\mu \nu} \partial^{\rho} \partial^{\sigma} \bar{h}_{\rho \sigma}\right)= \\
& -\theta^{\alpha \beta} \theta_{\mu \gamma}\left(\partial_{\nu} \partial_{\alpha} h_{\beta}{ }^{\gamma}-\partial^{\gamma} \partial_{\alpha} h_{\beta \nu}\right)-\theta^{\alpha \beta} \theta_{\nu \gamma}\left(\partial_{\mu} \partial_{\alpha} h_{\beta}{ }^{\gamma}-\partial^{\gamma} \partial_{\alpha} h_{\beta \mu}\right)-2 \eta_{\mu \nu} \theta^{\alpha \beta} \theta^{\rho}{ }_{\sigma} \partial_{\rho} \partial_{\alpha} h_{\beta}{ }^{\sigma} .
\end{aligned}
$$

We have also to consider the equation coming from (4.3). Since it is second order in $\theta$ there is no term with $\bar{h}$ and we get

$$
3 \theta^{\alpha \beta} \theta^{\rho \sigma} \partial_{\nu} \partial_{\rho} \partial_{\alpha} h_{\beta \sigma}+\theta^{\alpha \beta} \theta_{\nu \gamma}\left(\square \partial_{\alpha} h_{\beta}^{\gamma}-\partial^{\gamma} \partial^{\mu} \partial_{\alpha} h_{\beta \mu}\right)=0 .
$$

Using the field equation for $h_{\mu \nu}$ it reduces to

$$
\theta^{\alpha \beta} \theta^{\rho \sigma} \partial_{\nu} \partial_{\rho} \partial_{\alpha} h_{\beta \sigma}=0 .
$$

For $\nu=0$ the equation is trivial while for $\nu \neq 0$ (5.11) yields

$$
\theta^{0 j} \theta^{0 k} \partial_{i} \partial_{j} \partial_{k} h_{00}+\theta^{j m} \theta^{k n} \partial_{i} \partial_{j} \partial_{k} h_{m n}=0 .
$$

Introducing the vector notation $\theta^{0 i}=\tilde{\theta}^{i}, \quad \theta^{i j}=\epsilon^{i j k} \theta^{k}$, we find the solution of (15.12) to be

$$
\overrightarrow{\tilde{\theta}}= \pm \vec{\theta} .
$$

Then the time-space components of $\theta^{\mu \nu}$ are proportional to the space-space components. This is to be contrasted with the situation of noncommutative quantum field theories in flat spacetime. There the theories with time-space components have troubles with causality. This happens because there is no decoupling limit in string theory which generates a background with a B-field which has different electric and magnetic components which are perpendicular to each other [4]. In our case, the string theory background has a B-field with electric and magnetic components which are parallel or antiparallel. In this case the same happens, no decoupling limit does exist [21]. However, we are now in curved spacetime and closed strings need no longer be decoupled.

We can then go back to (5.9) and solve it. We get

$$
\begin{aligned}
& \bar{h}_{00}=3 G \vec{\theta}^{2} h-G(\vec{n} \cdot \vec{\theta})^{2} h+\alpha \vec{\theta}^{2} h, \\
& \bar{h}_{0 i}=0, \\
& \bar{h}_{i j}=G \delta^{i j}\left(\vec{\theta}^{2}-(\vec{n} \cdot \vec{\theta})^{2}\right) h+G\left(\theta^{i} n^{j}+\theta^{j} n^{i}\right)(\vec{n} \cdot \vec{\theta}) h+\alpha n^{i} n^{j} \vec{\theta}^{2} h .
\end{aligned}
$$

The $\alpha$ dependent terms are proportional to the homogeneous solution (5.8) and can be absorbed in it so we set $\alpha$ to zero. Then the noncommutative contribution to the metric at the linearized level is

$$
\begin{aligned}
g_{00} & =1+\left(1+3 G \vec{\theta}^{2}-G(\vec{n} \cdot \vec{\theta})^{2}\right) h, \\
g_{0 i} & =0, \\
g_{i j} & =-\delta^{i j}+\left[n^{i} n^{j}+G \delta^{i j}\left(\vec{\theta}^{2}-(\vec{n} \cdot \vec{\theta})^{2}\right)+G\left(\theta^{i} n^{j}+\theta^{j} n^{i}\right) \vec{n} \cdot \vec{\theta}\right] h .
\end{aligned}
$$


The effect of noncommutativity on a test particle can be seen by analyzing the geodesic equation. As usual, the time component equation relates the particle proper-time $\tau$ with the time $x^{0}=t$

$$
\frac{d t}{d \tau}=1-h_{00}-\bar{h}_{00}
$$

Then disregarding terms quadratic in the velocity we read off the the potential from the space components of the geodesic equation

$$
\frac{d^{2} x^{i}}{d t^{2}}=-\frac{1}{2} \partial_{i}\left[h+G\left(3 \vec{\theta}^{2}-(\vec{n} \cdot \vec{\theta})^{2}\right) h\right] .
$$

The Newtonian potential has a noncommutative contribution proportional to $\vec{\theta}^{2}$ which can be regarded as giving rise to an effective Newton constant $G\left(1+\frac{3}{2} G \overrightarrow{\theta^{2}}\right)$. It can be larger or smaller than $G$ (recall that in the action (4.1) we can set $\theta^{2} \rightarrow-\theta^{2}$ ). The angular dependent piece $(\vec{n} \cdot \vec{\theta})^{2}$ also contributes to the potential. The force on a test particle is given by

$$
\frac{d^{2} x^{i}}{d t^{2}}=\left[n^{i}+3 G\left(\vec{\theta}^{2}-(\vec{n} \cdot \vec{\theta})^{2}\right) n^{i}+2 G(\vec{n} \cdot \vec{\theta}) \theta^{i}\right] \frac{h}{2 r} .
$$

Even though the term proportional to $\vec{\theta}^{2}-(\vec{n} \cdot \vec{\theta})^{2}$ looks like a dipole the force still goes like $1 / r^{2}$ and is radial. This radial correction has a strength given by the effective Newton constant discussed before. And the term proportional to $\vec{\theta}$ produces in general a force off the plane of the orbit and is also periodic for closed orbits. Notice that if $\vec{\theta}$ is perpendicular to the plane of the orbit then no periodic effect due to noncommutativity is seen. A better treatment, which could be confronted which experimental data, would require to keep general relativity corrections which were disregarded in this approximation.

\section{CONCLUSION}

We have shown how we can generalize properties of the noncommutative parameter $\theta^{\mu \nu}$ from flat spacetime to curved spacetime in the simplest way possible. It can regarded as a covariantly constant tensor or as satisfying (2.9). In the case of a covariantly constant $\theta^{\mu \nu}$ general diffeomorphisms reduce to volume preserving transformations which also preserve $\theta^{\mu \nu}$. This gives a natural explanation for this symmetry which was found in [12].

Simple extensions of the Moyal product or the Kontsevich product to curved spacetime lead to nonassociative products. Maybe this is related to the findings of [13] where a structure which can not be written in terms of the Moyal product was detected. This means that more general deformations, may be along the lines of [23], must be considered.

Another interesting aspect is that the propagator in curved spacetime now depends on $\theta$ in contradistinction with the flat spacetime case where propagators receive no noncommutative contributions.

In the case of a covariantly constant $\theta^{\mu \nu}$ general relativity reduces to unimodular gravity. In the linearized limit we found a constraint between the time-space and space-space components of $\theta^{\mu \nu}$. We then obtained the Newtonian potential in unimodular gravity and its noncommutative correction. It still depends on $1 / r$ but has an effective Newton constant and an angular dependent piece. We have worked in the linearized approximation and general relativity contributions should also be taken into account to be able to confront with experiments. Cosmological corrections due to noncommutativity may also be relevant. Studies in these directions are being developed. 


\section{ACKNOWLEDGMENTS}

E.H. is supported by FAPESP through grant 03/09044-9. V.O.R. is partially supported by CNPq, FAPESP and PRONEX under contract CNPq 66.2002/1998-99. V.O.R. also thanks conversations with Álvaro Restuccia which pointed out reference [23].

[1] H. S. Snyder, "Quantized Space-Time," Phys. Rev. 71, 38 (1947).

[2] N. Seiberg and E. Witten, "String theory and noncommutative geometry," JHEP 9909, 032 (1999) arXiv:hep-th/9908142.

[3] For a review see: M. R. Douglas and N. A. Nekrasov, "Noncommutative field theory," Rev. Mod. Phys. 73, 977 (2001) arXiv:hep-th/0106048; R. J. Szabo, "Quantum field theory on noncommutative spaces," Phys. Rept. 378, 207 (2003) arXiv:hep-th/0109162; V. O. Rivelles, "Supersymmetry and gravity in noncommutative field theories," Nucl. Phys. Proc. Suppl. 127, 63 (2004) arXiv:hep-th/0305122.

[4] N. Seiberg, L. Susskind and N. Toumbas, "Space/time non-commutativity and causality," JHEP 0006, 044 (2000) arXiv:hep-th/0005015.

[5] S. Minwalla, M. Van Raamsdonk and N. Seiberg, "Noncommutative perturbative dynamics," JHEP 0002, 020 (2000) arXiv:hep-th/9912072.

[6] V. O. Rivelles, "Noncommutative field theories and gravity," Phys. Lett. B 558, 191 (2003) arXiv:hep-th/0212262.

[7] For a review see: R. J. Szabo, "Symmetry, gravity and noncommutativity," arXiv:hep-th/0606233

[8] A. H. Chamseddine, G. Felder and J. Frohlich, "Gravity in noncommutative geometry," Commun. Math. Phys. 155, 205 (1993) arXiv:hep-th/9209044; ;. Madore and J. Mourad, "A Noncommutative extension of gravity," Int. J. Mod. Phys. D 3, 221 (1994) arXiv:gr-qc/9307030; A. Jevicki and S. Ramgoolam, "Non-commutative gravity from the AdS/CFT correspondence," JHEP 9904, 032 (1999) arXiv:hep-th/9902059; J. W. Moffat, "Noncommutative quantum gravity," Phys. Lett. B 491, 345 (2000) arXiv:hep-th/0007181; S. Cacciatori, D. Klemm, L. Martucci and D. Zanon, "Noncommutative Einstein-AdS gravity in three dimensions," Phys. Lett. B 536, 101 (2002) arXiv:hep-th/0201103; S. Cacciatori, A. H. Chamseddine, D. Klemm, L. Martucci, W. A. Sabra and D. Zanon, "Noncommutative gravity in two dimensions," Class. Quant. Grav. 19, 4029 (2002) arXiv:hep-th/0203038; M. A. Cardella and D. Zanon, "Noncommutative deformation of four dimensional Einstein gravity," Class. Quant. Grav. 20, L95 (2003) arXiv:hep-th/0212071; H. Garcia-Compean, O. Obregon, C. Ramirez and M. Sabido, "Noncommutative self-dual gravity," Phys. Rev. D 68, 044015 (2003) arXiv:hep-th/0302180; J. M. Romero and J. D. Vergara, "The Kepler problem and non commutativity," Mod. Phys. Lett. A 18, 1673 (2003) arXiv:hep-th/0303064; D. V. Vassilevich, "Quantum noncommutative gravity in two dimensions," Nucl. Phys. B 715, 695 (2005) arXiv:hep-th/0406163; P. Nicolini, "A model of radiating black hole in noncommutative geometry," J. Phys. A 38, L631 (2005) arXiv:hep-th/0507266; P. Nicolini, A. Smailagic and E. Spallucci, "Noncommutative geometry inspired Schwarzschild black hole," Phys. Lett. B 632, 547 (2006) arXiv:gr-qc/0510112, C. Deliduman, "Noncommutative Gravity in Six Dimensions," arXiv:hep-th/0607096

[9] A. H. Chamseddine, "Complexified gravity in noncommutative spaces," Commun. Math. Phys. 
218, 283 (2001) arXiv:hep-th/0005222 ; A. H. Chamseddine, "Deforming Einstein's gravity," Phys. Lett. B 504, 33 (2001) arXiv:hep-th/0009153 ; H. Nishino and S. Rajpoot, "Teleparallel complex gravity as foundation for noncommutative gravity," Phys. Lett. B 532, 334 (2002) arXiv:hep-th/0107216 ; A. H. Chamseddine, "Invariant actions for noncommutative gravity," J. Math. Phys. 44, 2534 (2003) arXiv:hep-th/0202137; A. H. Chamseddine, "Sl(2,c) gravity with complex vierbein and its noncommutative extension," Phys. Rev. D 69, 024015 (2004) arXiv:hep-th/0309166.

[10] P. Aschieri, C. Blohmann, M. Dimitrijevic, F. Meyer, P. Schupp and J. Wess, "A gravity theory on noncommutative spaces," Class. Quant. Grav. 22, 3511 (2005) arXiv:hep-th/0504183; P. Aschieri, M. Dimitrijevic, F. Meyer and J. Wess, "Noncommutative geometry and gravity," Class. Quant. Grav. 23, 1883 (2006) arXiv:hep-th/0510059; F. Meyer, "Noncommutative spaces and gravity," arXiv:hep-th/0510188 B. M. Zupnik, "Reality in noncommutative gravity," arXiv:hep-th/0512231 A. P. Balachandran, T. R. Govindarajan, K. S. Gupta and S. Kurkcuoglu, "Noncommutative two dimensional gravities," arXiv:hep-th/0602265 A. Kobakhidze, "Theta-twisted gravity," arXiv:hep-th/0603132 S. Kurkcuoglu and C. Saemann, "Drinfeld Twist And General Relativity With Fuzzy Spaces," arXiv:hep-th/0606197.

[11] For earlier references see: E. Alvarez, "Can one tell Einstein's unimodular theory from Einstein's general relativity?," JHEP 0503, 002 (2005) arXiv:hep-th/0501146.

[12] X. Calmet and A. Kobakhidze, "Noncommutative general relativity," Phys. Rev. D 72, 045010 (2005) arXiv:hep-th/0506157; X. Calmet, "Cosmological constant and noncommutative spacetime," arXiv:hep-th/0510165 X. Calmet and A. Kobakhidze, "Second Order Noncommutative Corrections to Gravity," arXiv:hep-th/0605275. P. Mukherjee and A. Saha, "Comment on the first order noncommutative correction to gravity," arXiv:hep-th/0605287.

[13] L. Alvarez-Gaume, F. Meyer and M. A. Vazquez-Mozo, "Comments on noncommutative gravity," arXiv:hep-th/0605113.

[14] A covariantly constant $\theta^{\mu \nu}$ has been used to investigate phenomenological consequences of noncommutativity in cosmology in F. Lizzi, G. Mangano, G. Miele and M. Peloso, "Cosmological perturbations and short distance physics from noncommutative geometry," JHEP 0206, 049 (2002) arXiv:hep-th/0203099.

[15] R. Jackiw, S. Y. Pi and A. P. Polychronakos, "Noncommuting gauge fields as a Lagrange fluid," Annals Phys. 301, 157 (2002) arXiv:hep-th/0206014.

[16] M. Chaichian, M. N. Mnatsakanova, K. Nishijima, A. Tureanu and Y. S. Vernov, "Towards an axiomatic formulation of noncommutative quantum field theory," arXiv:hep-th/0402212.

[17] M. Kontsevich, "Deformation quantization of Poisson manifolds, I," Lett. Math. Phys. 66, 157 (2003) arXiv:q-alg/9709040.

[18] A. Das and J. Frenkel, "Kontsevich product and gauge invariance," Phys. Rev. D 69, 065017 (2004) arXiv:hep-th/0311243.

[19] L. Cornalba and R. Schiappa, "Nonassociative Star Product Deformations For DBrane Worldvolumes In Curved Backgrounds," Commun. Math. Phys. 225, 33 (2002) arXiv:hep-th/0101219.

[20] J. J. van der Bij, H. van Dam and Y. J. Ng, "Theory Of Gravity And The Cosmological Term: The Little Group Viewpoint," Physica 116A, 307 (1982).

[21] O. Aharony, J. Gomis and T. Mehen, "On Theories With Light-Like Noncommutativity," JHEP 0009, 023 (2000) arXiv:hep-th/0006236.

[22] D. R. Finkelstein, A. A. Galiautdinov and J. E. Baugh, "Unimodular relativity and cosmological constant," J. Math. Phys. 42, 340 (2001) arXiv:gr-qc/0009099. 
[23] B. V. Fedosov, "A simple geometrical construction of deformation quantization," J. Diff. Geom. 40, 213 (1994). 